\title{
ON THE FINITENESS OF THE PROJECTIVE DIMENSION OF CERTAIN IDEALS
}

\author{
ANTONIO G. RODICIO
}

(Communicated by Louis J. Ratliff, Jr.)

\begin{abstract}
For a local noetherian ring, a subset of a minimal system of generators of the maximal ideal is a regular sequence if the ideal it generates has finite projective dimension.
\end{abstract}

We prove

THEOREM. Let $(A, m, K)$ be a local noetherian ring and $\left\{x_{1}, \ldots, x_{n}\right\}$ a minimal set of generators for $m$. If the ideal of $A$ generated by $x_{1}, \ldots, x_{r}(r \leq n)$ has finite projective dimension, then $x_{1}, \ldots, x_{r}$ is a regular sequence.

For $r=n$ we obtain the Serre's theorem: a local noetherian ring of finite global dimension is regular. We also obtain the well-known result: each subset of a regular system of parameters of a regular local ring is a regular sequence.

To prove the theorem we use

LEMMA. Let $(A, m, K)$ be a local noetherian ring and $x$ a nonzero divisor in $A$ which is not contained in $\mathrm{m}^{2}$. Let $N$ be a finitely generated $A /(x)$-module. If $N$ has finite projective dimension as an $A$-module, then $N$ has finite projective as an $A /(x)$-module.

PrOOF. Since each free $A /(x)$-module has projective dimension one as an $A$ module, it suffices to consider that $N$ has projective dimension one as an $A$-module. Let $0 \rightarrow R \rightarrow F \rightarrow N \rightarrow 0$ be an exact sequence with $R \subset m F, R$ and $F$ being free $A$-modules. Let $\left\{b_{1}, \ldots, b_{n}\right\}$ be a basis of $F$. The elements $x b_{1}, \ldots, x b_{n}$ are in $R$ and their classes $\bmod m R$ are $k$-linearly independent. Hence $\left\{x b_{1}, \ldots, x b_{n}\right\}$ is a basis of $R$, since the rank of $R$ cannot be larger than the rank of $F$. Therefore $N$ is a free $A /(x)$-module.

We give now an alternative proof of the lemma, which is more complicated, but it provides a formula for relating the Betti numbers of $N$ relative to $A$ and $A /(x)$. We shall show that there exists an isomorphism

$$
\operatorname{Tor}_{n+1}^{A}(N, K) \simeq \operatorname{Tor}_{n+1}^{A /(x)}(N, K) \oplus \operatorname{Tor}_{n}^{A /(x)}(N, K) \quad \text { for } n \geq 1
$$

We consider the change-rings spectral sequence

$$
E_{p, q}^{2}=\operatorname{Tor}_{p}^{A /(x)}\left(N, \operatorname{Tor}_{q}^{A}(A /(x), m)\right) \Rightarrow \operatorname{Tor}_{p+q}^{A}(N, m) .
$$

Received by the editors February 23, 1987 and, in revised form, April 24, 1987. 1980 Mathematics Subject Classification (1985 Revision). Primary 13D05. 
Since $E_{p, 0}^{2}=\operatorname{Tor}_{p}^{A /(x)}(N, m / x m)$ and $E_{p, q}^{2}=0$ for $q>0$, the spectral sequence degenerates and we have isomorphisms $\operatorname{Tor}_{n}^{A /(x)}(N, m / x m) \simeq \operatorname{Tor}_{n}^{A}(N, m)$ for $n \geq$ 1. On the other hand there exists an isomorphism of $A /(x)$-modules $m / x m \simeq$ $m /(x) \oplus K\left[\mathbf{2}\right.$, Lemma 2]. Hence $\operatorname{Tor}_{n}^{A /(x)}(N, m /(x)) \oplus \operatorname{Tor}_{n}^{A}(N, K) \simeq \operatorname{Tor}_{n}^{A}(N, m)$ for $n \geq 1$ and the result follows.

PROOF OF THE THEOREM. Let $I$ be the ideal of $A$ generated by $x_{1}, \ldots, x_{r}$. It suffices to show that $I$ is generated by a regular sequence [1, Proposition 1.4.8]. Induction on $r$. Since $I$ has finite projective dimension, there exists a nonzero divisor in $I-m I$ [2, Lemma 3]. Choosing, if necessary, a new system of generators of $m$, we may assume that $x_{1}$ is a nonzero divisor. Therefore the lemma implies that $I /\left(x_{1}\right)$ has finite projective dimension as an $A /\left(x_{1}\right)$-module. By induction $x_{2}, \ldots, x_{r}$ is a regular sequence $\bmod \left(x_{1}\right)$. Then $x_{1}, \ldots, x_{r}$ is a regular sequence.

ACKNOWLEDGMENT. I am grateful to the referee for his helpful suggestions and the alternate proof of the lemma.

\section{REFERENCES}

1. T. H. Gulliksen and G. Levin, Homology of local rings, Queen's Papers in Pure and Appl. Math., no. 20, Queen's Univ., Kingston, Canada, 1969

2. W. V. Vasconcelos, Ideals generated by R-sequences, J. Algebra 6 (1967), 309-316.

Departamento de Algebra, Facultad de Matematicas, 15771 Santiago de COMPOSTELA, SPAiN 